\title{
Quantitative FFPE Histopathology of Wound Healing in Mice using Special Stains
}

\author{
Jordan Yaron
}

School for Engineering of Matter, Transport and Energy, Arizona State University, Tempe, Arizona, United States

Dermal wounds are a major health care burden. In the US, more than 6 million chronic wound cases amount to a cost of over $\$ 20$ billion per year and nearly $5 \%$ of the total cost of Medicare and Medicaid ${ }^{[1]}$. Dysregulation in the onset or resolution of any phase of repair (I - hemostasis, II - inflammation, III - proliferation, IV remodeling) leads to impaired healing and contributes to increased morbidity and mortality in common comorbidities such as diabetes ${ }^{[2]}$. Despite intense investigation, there remains a significant, unmet need for improved approaches to enhance the wound healing process.

The use of rodent models of wound healing, especially the splinted full-thickness excisional model, which requires second intention/granulation mechanisms ${ }^{[3]}$, allows for rapid and reproducible preclinical assessment of investigational therapies in strains with relevant physiological properties (e.g., db/db diabetic mice) or genetic knockouts. However, several properties of mouse skin introduce technical challenges for histopathology. First, adult mouse skin is approximately $1 \mathrm{~mm}$ thick, 4-5 times thinner than human or porcine skin. Accordingly, care should be given to keep the skin flattened during fixation to avoid artifact caused by curling. This is usually achieved by placing the skin specimen between two pieces of tissue embedding sponges in a cassette. Second, between the epidermis and the hypodermis, skin is predominantly composed of connective tissue with only a sparse interspersing of immune cells, fibroblasts, blood vessels, sensory neurons and hair follicles - but no formal parenchymal tissue. Consequently, floated sections are prone to overexpansion, sections weakly attach to slides and connective tissue integrity and architecture can be unacceptably damaged by heat-induced epitope retrieval for immunohistochemistry (IHC). We have found that floating single sections (instead of ribbons), "sandwiching" sections with a second glass slide secured with a clip, and overnight incubation in retrieval buffer at $60^{\circ} \mathrm{C}$ largely resolves these issues ${ }^{[4,5]}$, albeit at the cost of time and throughput.

Notwithstanding these challenges, an advantage of investigating wound healing from a histochemical approach is the availability of numerous special stains which can highlight key biological mechanisms in healing, while also avoiding the potential artifact-inducing steps and limited throughput of IHC. Combining the patterns of these special stains with the ImageJ/FIJI package affords a convenient, low-cost way to perform quantitative analyses on tissues ${ }^{[6]}$.

A key example of the utility of special stains in wound healing is the study of collagen. Type I collagen in granulation tissue deposited by fibroblasts is an essential, early scaffold required to fill the wound bed and allow invasion of immune cells and angiogenic precursors ${ }^{[7,8]}$. Later, maturation to a primarily type III collagen permits denser angiogenic sprouting and structural support for more thoroughly differentiated vessels and sensory neurons. The most common special stain for studying collagen in wound healing is Masson's Trichrome (Fig 1A), which results in black-stained nuclei, red-stained muscle and keratin and blue-stained collagen, which appears more vibrant with denser deposition. Usually, the optical density of the blue-stained area (which can be isolated using the Colour Deconvolution method in ImageJ/FIJI ${ }^{[9]}$ ) is measured and used as a metric of the degree of collagen deposition. We have also found that higher complexity analyses can be performed using other plugins such as LocalThickness or Directionality to compare the bundling and organization of healed skin to intact, healthy $\operatorname{skin}^{[5,10,11]}$. The second most common special stain for collagen in wound healing studies is Picrosirius Red (PR; Fig 1B), which will stain collagen bright red with staining intensity related to the degree of deposition. An additional advantage of PR is the ability to determine bundle orientation through intrinsic birefringence of the stain when viewed through circularly polarized light. It should 
be noted that despite the previously attribution of the polarized green staining as type III collagen and polarized red staining as type I collagen, it has been clearly shown that the birefringence is affected only by the molecular order and orientation - metrics which are nonetheless useful for investigating wound healing ${ }^{[12]}$. A third, but comparatively uncommon, special stain for collagen is Herovici's Polychrome (Fig 1C), which exhibits higher fidelity staining for type III collagen (blue) and type I collagen (red/pink) versus $\mathrm{PR}^{[13]}$. Color deconvolution in ImageJ/FIJI and ratiometric quantification of type I versus type III collagen in what we term the "Herovici Ratio" can provide a useful metric to compare healing efficacies of different treatments ${ }^{[4]}$.

Other special stains also have distinct utility in wound healing. For example, toluidine blue (Fig 1D) is a metachromatic stain which results in blue-stained glycosamino- and proteoglycans and vividly and specifically purple-stained mast cells, due to abundant heparin and histamine in their granules. Mast cells have key roles in wound healing ${ }^{[14]}$ and their rapid and simple identification by a special stain, which can be easily identified using the ImageJ/FIJI plugins Colour Deconvolution and Analyze Particles, further combined with IHC techniques to multiplex mast cells with other tissue markers, provides a simple strategy for increasing analytical depth.

Special stains are (i) generally more rapid protocols than IHC, (ii) use comparatively low-cost reagents, (iii) do not require high temperature treatments which can destroy the quality of sections, and (iv) can be multiplexed and quantitatively analyzed using widely available and simple to use software packages. These properties highlight the utility of special stains in any histochemical workflow, and particularly in the investigation of dermal wound healing.

A

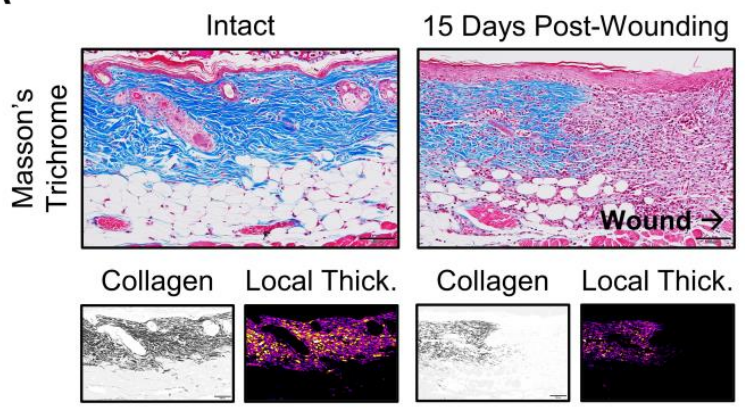

C



B

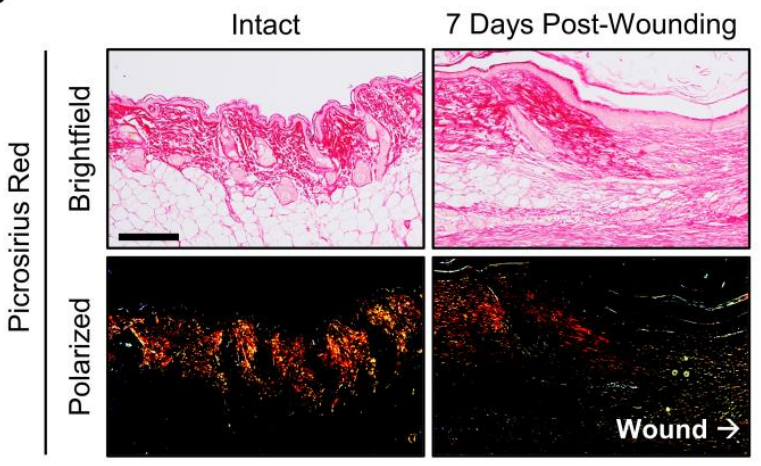

a. Toluidine Blue



Figure 1. Special stains for investigating dermal wound healing. (A) Masson's Trichrome is a common stain for collagen, which can be accurately color deconvolved to perform more complex texture analyses, 
such as local bundle thickness. (B) Picrosirius Red stains collagen vivid red, but can be further analyzed by circularly polarized light to reveal collagen bundle order and organization by red and green birefringence. (C) Herovici's Polychrome stains Type I and Type III collagen with sufficient fidelity to observe granulation tissue maturation over time. (D) Toluidine blue metachromatically stains mast cells a vivid purple with high specificity which can allow automatic identification and quantification of mast cells in tissues by color deconvolution and particle analysis. Scale bars: $\mathrm{A}, \mathrm{C}=100$ microns, $\mathrm{B}=200$ microns.

\section{References}

[1] Y. Niu, Q. Li, Y. Ding, L. Dong, C. Wang, Adv. Drug Deliv. Rev. 2019, 146, 190.

[2] A. J. Singer, R. A. F. Clark, N. Engl. J. Med. 1999, 341, 738.

[3] R. D. Galiano, J. Michaels V, M. Dobryansky, J. P. Levine, G. C. Gurtner, Wound Repair Regen. 2004, 12, 485.

[4] J. R. Yaron, L. Zhang, Q. Guo, E. A. Awo, M. Burgin, L. N. Schutz, N. Zhang, J. Kilbourne, J. Daggett-vondras, K. M. Lowe, A. R. Lucas, Pharmaceutics 2020, 12, 1003.

[5] L. Zhang, J. R. Yaron, A. M. Tafoya, S. E. Wallace, J. Kilbourne, S. Haydel, K. Rege, G. McFadden, A. R. Lucas, J. Clin. Med. 2019, 8, 1626.

[6] J. Schindelin, I. Arganda-Carreras, E. Frise, V. Kaynig, M. Longair, T. Pietzsch, S. Preibisch, C. Rueden, S. Saalfeld, B. Schmid, J.-Y. Tinevez, D. J. White, V. Hartenstein, K. Eliceiri, P. Tomancak, A. Cardona, Nat. Methods 2012, 9, 676.

[7] X. Feng, M. G. Tonnesen, S. A. Mousa, R. A. F. Clark, Int. J. Cell Biol. 2013, 2013, DOI $10.1155 / 2013 / 231279$.

[8] B. P. O’Rourke, A. H. Kramer, L. L. Cao, M. Inayathullah, H. Guzik, J. Rajadas, J. D. Nosanchuk, D. J. Sharp, Adv. Wound Care 2019, 8, 91.

[9] A. C. Ruifrok, D. A. Johnston, Anal. Quant. Cytol. Histol. 2001, 23, 291.

[10] T. Saito, J.-I. Toriwaki, Pattern Recognit. 1994, 27, 1551.

[11] T. Hildebrand, P. Rüegsegger, J. Microsc. 1997, 185, 67.

[12] R. Lattouf, R. Younes, D. Lutomski, N. Naaman, G. Godeau, K. Senni, S. Changotade, J. Histochem. Cytochem. 2014, 62, 751.

[13] M. Levame, F. Meyer, Pathol. Biol. (Paris). 1987, 35, 1183.

[14] B. C. Wulff, T. A. Wilgus, Exp. Dermatol. 2013, 22, 507. 